\title{
Conhecimento das puérperas sobre o plano de parto em um município do oeste do Paraná
}

\author{
Knowledge of postpartum women about the birth plan in a city in western Paraná
}

Conocimiento de las puérperas sobre el plan de parto en una ciudad del occidente de Paraná

Camila Wiggers $^{1 *}$, Michelli de Araujo ${ }^{1}$, Wesley Martins ${ }^{1}$, Cinthya de Fátima Oliveira Strada ${ }^{1}$.

\section{RESUMO}

Objetivo: Identificar a experiência e as dificuldades em relação ao Plano de Parto (PP) das puérperas que frequentam uma UBS de um município do oeste do Paraná. Métodos: Trata-se de uma pesquisa descritiva exploratória de caráter quantiqualitativa, realizada com 13 puérperas de uma unidade de saúde, por meio de um instrumento semiestruturado. Resultados: A análise dos dados demonstrou que $69 \%$ das puérperas não conheciam o plano de parto, a sua não utilização está relacionada ao não conhecimento dessa ferramenta por elas e pelos profissionais de saúde, falta de apoio e incentivo profissional, havendo muitas lacunas e desafios a serem enfrentados apesar dos inúmeros efeitos benéficos do plano de parto. Todas as participantes indicaram que a elaboração do plano é de suma importância para sanar dúvidas, medos, sofrimentos, reduzir intervenções desnecessárias e obter mais segurança. Conclusão: Foi constatado que pouca é a divulgação do PP, existindo muitos bloqueios e desafios a serem superados e que os profissionais da saúde precisam desenvolver essa prática, visto que a enfermagem tem um papel muito importante nessa etapa, pois se a gestante receber estímulo da equipe de saúde desde o início do pré-natal poderá optar por escolhas que a aproximem de um atendimento com resultados mais satisfatórios.

Palavras-chave: Plano de parto, Parto, Gestantes.

\section{ABSTRACT}

Objective: To identify the experience and difficulties in relation to the Birth Plan (PP) of mothers attending a UBS in a municipality in western Paraná. Methods: This is exploratory descriptive research of quantiqualitative character, carried out with 13 postpartum women from a health unit, using a semi-structured instrument. Results: Data analysis showed that $69 \%$ of postpartum women did not know the birth plan, its non-use is related to lack of knowledge of this tool by them and by health professionals, lack of professional support and encouragement, with many gaps and challenges to be faced despite the numerous beneficial effects of the birth plan. All participants indicated that the elaboration of the plan is of paramount importance to resolve doubts, fears, suffering, reduce unnecessary interventions and obtain more security. Conclusion: It was found that little is the dissemination of the PP, there are many obstacles and challenges to be overcome and that health professionals need to develop this practice, as nursing has a very important role in this stage, because if the pregnant woman receives encouragement from Since the beginning of prenatal care, the health team will be able to choose choices that bring them closer to care with more satisfactory results.

Key words: Delivery plan, Delivery, Pregnant women.

\section{RESUMEN}

Objetivo: Identificar la experiencia y dificultades en relación al Plan de Parto (PP) de madres que asisten a una Unidad Básica de Salud en un municipio del occidente de Paraná. Métodos: Se trata de una investigación descriptiva exploratoria de carácter cuanti-cualitativo, realizada con 13 puérperas de una UBS, utilizando un instrumento semiestructurado. Resultados: El análisis de datos mostró que el $69 \%$ de las puérperas no conocía el plan de parto, su no uso se relaciona con el desconocimiento de esta herramienta por parte de ellas y de los profesionales de la salud, falta de apoyo y estímulo profesional, con muchas brechas y desafíos.

${ }^{1}$ Centro Universitário Dinâmica das Cataratas (UDC), Foz do lguaçu - PR.

*E-mail: wiggers.enfer@hotmail.com

SUBMETIDO EM: 11/2021

| ACEITO EM: 11/2021

PUBLICADO EM: 11/2021 
a afrontar a pesar de los numerosos efectos beneficiosos del plan de parto. Todos los participantes indicaron que la elaboración del plan es de suma importancia para resolver dudas, miedos, sufrimientos, reducir intervenciones innecesarias y obtener más seguridad. Conclusión: Se encontró que poca es la difusión de la $\mathrm{PP}$, hay muchos obstáculos y desafíos a superar y que los profesionales de la salud necesitan desarrollar esta práctica, ya que la enfermería tiene un papel muy importante en esta etapa, porque si la gestante recibe estímulo Desde el inicio de la atención prenatal, el equipo de salud podrá elegir opciones que las acerquen a la atención con resultados más satisfactorios.

Palabras clave: Plan de parto, Parto, Embarazadas.

\section{INTRODUÇÃO}

O Plano de Parto (PP), de acordo com Santana WN (2020), é um documento estilo carta de intenções na qual a gestante declara qual é o atendimento que espera para si e para o seu bebê, durante o processo de nascimento. A construção do Plano de Parto geralmente acontece durante o pré-natal. Sua aplicação permite que os profissionais da assistência e as gestantes apropriem-se previamente dos procedimentos que elas gostariam que ocorressem durante o nascimento do seu filho. Dessa forma, permite que os profissionais respeitem as decisões da mulher e garantam uma assistência de acordo com suas vontades, contribuindo para o empoderamento feminino e a promoção da saúde materna e neonatal.

Quando o parto passou a ser realizado no ambiente hospitalar ele era visto como algo patológico e que necessitava de medicalização, para Sheila Kitzinger o parto é algo fisiológico que irá ocorrer com a maioria das mulheres, sendo então realizado por ela o primeiro modelo de plano de parto em 1980, a partir daí Estados Unidos e Canadá deram início ao seu uso (CHACHAM AS,1996 apud SANTOS FSR, et al., 2019).

Diante disso, o uso do PP é recomendado pela Organização Mundial da Saúde (OMS) desde 1986 e pelo Ministério da Saúde no Brasil após a implantação do Programa Rede Cegonha, em 2011. No plano de parto, as gestantes expressam suas vontades e desejos durante o seu trabalho de parto, parto e pós-parto, que incluem desde a ingesta alimentar e hídrica, a posição na hora de parir, uso de medicamentos e procedimentos sem real necessidade, orientações sobre tudo que irá ser realizado (SANTANA WN, 2020).

Os benefícios do plano de parto são inúmeros, sendo eles; transferência de segurança as gestantes, participação ativa, apoio emocional, que diminuem a dor e tensão do trabalho de parto, dando segurança também aos profissionais por mostrar os detalhes e escolhas das mulheres gerando maior atenção e vínculo entre a gestante e os profissionais, atendimento mais personalizado (MOUTA RJO, 2017). Complementando isso, Cortés MS, et al. (2015), apontam que o PP auxilia na autonomia, segurança, eficiência e satisfação das parturientes no momento do parto.

Em vista disso entende-se que a gestante possui várias opções para acrescentar em seu plano de parto, que trazem muitos benefícios, as opções são; atender demandas na hora do parto (como oferecer agua, permitir a deambulação, direito a acompanhante), permitir que a mulher fique na posição que achar mais confortável, respeitar as escolhas sobre métodos para analgesia (seja meios naturais, massagens, ou até anestesia, a escolha é dela), evitar procedimentos desnecessários na parturiente (episiotomia, uso de ocitocina para estimular o parto, lavagem intestinal, tricotomia, jejum), evitar procedimentos desnecessários no bebe, permitir a permanência da doula e obstetra, deixar a mãe e filho juntos após o parto, cordão umbilical (esperar um tempo para que seja cortado), aguardar a mulher entrar em trabalho de parto. A mulher acaba adquirindo um maior respeito ao apresentar o seu plano de parto, pois mostra que ela buscou conhecimento para este momento e vai lutar pelos seus direitos e por um parto digno (TORRES KN e ABI RACHED CD, 2017).

O pré-natal realizado pelo enfermeiro é uma etapa crucial e de muita importância na gestação, haja visto que ele é responsável por uma assistência integral, de qualidade, comunicativa, que garante cobertura, acompanhamento e acolhimento das gestantes (SILVA ALNV, et al., 2017). Todavia, mesmo sendo reconhecido há muitos anos, o plano de parto ainda é desconhecido por profissionais de saúde, bem como em hospitais e maternidades, fazendo com que seu uso não aconteça (MOUTA RJO, 2017). 
De acordo com uma pesquisa realizada por Rosa ECS (2020), que foi constituída de profissionais que trabalham com gestantes num hospital universitário do interior de São Paulo, analisou-se à relação ao cumprimento do plano de parto, $65 \%$ dos profissionais relatam seguir o plano, caso a gestante o apresente, o restante, $35 \%$, informaram que não seguem. Entretanto $80 \%$ relataram saber o que é o PP, porém apenas $20 \%$ obtiveram alguma informação sobre plano de parto pelo hospital.

Essa deficiência é explicada pela falta de estudos desenvolvidos sobre esse tema em nosso país, como apontado por Silva ALNV, et al. (2017). Um trabalho realizado com 106 gestantes, no município de Belo Horizonte - MG, verificou que $74 \%$ delas não receberam quaisquer informações sobre o plano de parto no momento do pré-natal, embora sua caderneta de gestante constasse o plano de parto, o que se evidencia a pouca valorização desse instrumento pelos profissionais na atenção básica (SILVA SG, et al., 2015).

A portaria № 569 , de $1^{\circ}$ de junho de 2000 do Ministério da Saúde, nos diz que as unidades básicas de saúde devem receber com dignidade o recém-nascido, a puérpera e sua família, já que realizar a assistência obstétrica e neonatal é um acompanhamento indispensável e muito importante nas unidades que inclui práticas seguras, humanizadas, organização e correto acolhimento e a não realização de intervenções desnecessárias (MINISTÉRIO DA SAÚDE, 2000).

Diante do exposto, esse estudo objetivou identificar a experiência e as dificuldades em relação ao plano de parto das puérperas que frequentam uma Unidade Básica de Saúde de um município do oeste do Paraná.

\section{MÉTODOS}

Trata-se de uma pesquisa transversal, descritiva e exploratória, de caráter quanti-qualitativo realizado com puérperas usuárias de um serviço público de saúde, Unidade Básica de Saúde, que abrange também uma área de ocupação de um município do Paraná.

Coleta de dados foi realizada nos meses de agosto e setembro de 2021. Os sujeitos da pesquisa são 13 puérperas usuárias de uma Unidade de Saúde de um determinado distrito sanitário de um município da região oeste do Paraná. Critérios de inclusão foram puérperas com idade acima de 18 anos, cadastradas na área de abrangência da unidade de saúde. E os critérios de exclusão, mulheres com idade inferior a 18 anos, não pertencentes a área de abrangência da unidade de saúde.

As pesquisadoras elaboraram um instrumento de coleta de dados que permite o levantamento de dados referente a caracterização das participantes da pesquisa, tais como: idade, raça / cor, situação socioeconômica, estado civil, escolaridade e número de filhos. Também serão indagadas a respeito do tema geral do presente projeto de pesquisa, ou seja, sobre o conhecimento e/ou experiência na utilização do Plano de Parto. Nesse levantamento, pretendeu-se conhecer os gargalos que dificultaram a plena utilização do método e em quais situações saiu diferente do planejado, sendo posteriormente realizado uma análise estatística descritiva dos resultados.

Este estudo só teve início após a aprovação do Comitê de Ética em Pesquisa Envolvendo Seres Humanos (CEP), através do parecer $n^{\circ} 4.901 .634$ vinculado ao Conselho Nacional de Ética em Pesquisa (CONEP), respeitando todas as questões éticas e legais regidos nas resoluções CNS 466/2012 e CNS 510/2015, mantendo a integridade física e emocional, a dignidade e os interesses de todos os envolvidos na pesquisa. Todos os sujeitos participantes da pesquisa assinaram o Termo de Consentimento Livre e Esclarecido (TCLE) e receberam uma cópia do mesmo.

\section{RESULTADOS}

Participaram da pesquisa 13 puérperas de um determinado município da região oeste do Paraná, usuárias de uma Unidade de Saúde da região sul do município, território que abrange uma grande área de ocupação. Das 40 mulheres que estavam sendo acompanhadas na unidade de saúde, 27 não se enquadraram nos critérios de inclusão estabelecidos previamente na pesquisa, sendo escolhido essa Unidade de Saúde devido 
a caracterização de sua população, por serem uma classe com uma renda e escolaridade mais baixa, de grande vulnerabilidade, logo tendo pouco conhecimento sobre o PP, sendo possível verificar na Tabela 1 os dados referentes a caracterização das participantes.

Tabela 1 - Caracterização das participantes da pesquisa quanto a idade, cor e raça, renda familiar, número de filhos, estado civil e escolaridade.

\begin{tabular}{|c|c|c|}
\hline Variável & $\mathbf{N}$ & $\%$ \\
\hline \multicolumn{3}{|l|}{ Idade } \\
\hline 18 a 22 anos & 3 & 23 \\
\hline 23 a 27 anos & 3 & 23 \\
\hline 28 a 33 anos & 6 & 46 \\
\hline 34 a 38 anos & 1 & 8 \\
\hline \multicolumn{3}{|l|}{ Cor / Raça } \\
\hline Branca & 6 & 46 \\
\hline Parda & 7 & 54 \\
\hline \multicolumn{3}{|l|}{ Renda familiar } \\
\hline Sem renda & 1 & 8 \\
\hline Menos de um salario & 1 & 8 \\
\hline Um a dois salários & 9 & 69 \\
\hline Três a quatro salários & 2 & 15 \\
\hline \multicolumn{3}{|l|}{ Número de filhos } \\
\hline Um filho & 4 & 31 \\
\hline Dois filhos & 3 & 23 \\
\hline Três filhos & 5 & 38 \\
\hline Quatro filhos & 1 & 8 \\
\hline \multicolumn{3}{|l|}{ Estado civil } \\
\hline Solteira & 6 & 46 \\
\hline Casada & 7 & 54 \\
\hline \multicolumn{3}{|l|}{ Escolaridade } \\
\hline Fundamental I completo & 1 & 8 \\
\hline Fundamental II completo & 5 & 38 \\
\hline Ensino médio completo & 6 & 46 \\
\hline Superior incompleto & 1 & 8 \\
\hline
\end{tabular}

Fonte: Wiggers C, et al., 2021.

Quanto a idade, percebe-se que $46 \%$ das participantes estão na faixa etária entre 28 a 33 anos, seguido das faixas de 18 a 22 anos e 23 a 27 anos, com 23\% cada. Em relação a raça / cor, a maioria (54\%) são pardas e $46 \%$ brancas.

Sobre a idade, Silva SG, et al. (2015) mencionam em seu estudo sobre conhecimento do plano de parto em gestantes que a idade predominante foi de 20 a 24 anos, correspondendo a $31 \%$ da amostra e que a raça/cor parda foi a mais prevalente (56\%). A desigualdade social é um fator considerável em relação aos indicadores de saúde materna, o que reflete na condição de vida e acesso a recursos sociais entre os diversos grupos da sociedade.

Quando questionadas sobre a renda familiar, 85\% das entrevistas apontaram receber até dois salários mínimos, sendo que 38\% destas possuem em média três filhos. Ainda para Silva SG, et al. (2015), em seu estudo constataram que $60 \%$ das gestantes também recebiam até 2 salários mínimos, o que mostra relação positiva entre as pesquisas. Referente ao número de filhos, Mouta (2017) constatou que $82 \%$ das entrevistadas eram primíparas, contrastando com nossos resultados.

Acerca do estado civil das participantes do estudo, 54\% mencionaram ser casadas e $46 \%$ afirmaram ter concluído o ensino médio. Rodrigues MS (2017) em seu estudo constatou que $90 \%$ das mulheres são casadas 
e 40\% possuem o ensino médio. Em contrapartida, no estudo de Mouta RJO, (2017), 55\% das parturientes são solteiras e $55 \%$ com ensino médio.

As condições sociais maternas como a baixa escolaridade e menor nível socioeconômico, podem acarretar desfechos desfavoráveis no decorrer da gestação, devido à assistência ineficaz oferecida a elas por instituições e profissionais despreparados para oferta-las igualdade de acesso ao serviço de saúde, considerando a particularidade social e cultural de cada mulher.

Na Tabela 2, são apresentados os resultados quanto ao conhecimento das puérperas em relação ao que é o plano de parto, ao seu direito legal para realiza-lo, se elaboraram tal plano, se recomendariam ele e qual foi o tipo de parto realizado por elas.

Tabela 2 - Conhecimento das participantes quanto seu conhecimento em relação ao plano de parto, seu direito legal de realiza-lo, se houve elaboração, recomendação do mes mo e o tipo de parto realizado.

\begin{tabular}{lcc}
\hline Variável & N & $\%$ \\
\hline Conhecimento sobre o PP & 4 & 31 \\
\hline Conhecia & 9 & 69 \\
Não conhecia & & \\
\hline Conhecimento sobre o direito legal & 3 & 23 \\
\hline Conhecia & 10 & 77 \\
Não conhecia & 4 & 31 \\
\hline Elaboração do PP & 9 & 69 \\
\hline Elaborou & & \\
Não elaborou & 13 & \\
\hline Recomendação do PP & & 38 \\
\hline Recomendaria & 5 & 62 \\
\hline Tipo de parto realizado por elas & 8 & \\
\hline Parto Cesárea &
\end{tabular}

Fonte: Wiggers C, et al., 2021.

Quanto ao número total de participantes, $69 \%$ não conheciam o que era o plano de parto, sendo $77 \%$ nem imaginavam ser seu direito legal. No estudo de Silva SG, et al. (2015), 74\% das participantes relatam não ter conhecimento sobre o plano de parto. E para Santos MLD (2020), 88,7\% das participantes não conheciam o plano de parto.

Questionadas sobre a elaboração do plano de parto, nove mulheres (69\%) mencionaram não ter criado, todavia, após conhecerem seus benefícios, 100\% das participantes relataram recomendar o uso. Quando levantado o tipo de parto que tiveram, $62 \%$ das entrevistadas mencionaram ter tido parto normal.

A respeito dos dados acima mencionados, no estudo de Santos FSR, et al. (2019) foram encontrados resultados que contrastam nossos dados, visto que $60 \%$ de suas participantes relataram ter realizado o plano de parto, entretanto o trabalho de parto dessas mulheres que realizaram o PP, ocorreu $54 \%$ em hospital/maternidade particular e 33\% em maternidade do Sistema Único de Saúde e a principal via de parto se iguala, $61 \%$ parto vaginal.

Já no estudo de Córtes MS, et al. (2015), foram analisados 9.303 partos nos anos de 2011 e 2012, tendo realizado o PP em somente 2,6\% ( $n=240)$. Apesar do Ministério da Saúde ter publicado um modelo de plano de parto em 2012 o número total de PP diminuiu 0,5\% em 2012 em relação a 2011 em um hospital da Espanha.

Além dos dados acima citados, as entrevistadas foram indagadas quanto ao respeito por parte dos profissionais de saúde durante o pré-natal até o momento do parto, onde todas responderam satisfatoriamente, todavia, $15 \%$ mencionaram não terem seguido conforme o planejamento. Questionadas 
sobre os motivos, uma relatou alterações devido a um descolamento de placenta e outra por não ter conseguido laqueadura.

Após o conhecimento sobre o plano de parto, todas as participantes relataram ser de suma importância o instrumento no planejamento. Na maioria das respostas ressaltaram que provavelmente isso sanaria as dúvidas, medos e sofrimento desnecessário, aumentando o controle das mulheres sobre o processo de parturição. Suas expectativas em relação ao atendimento para o parto constituem basicamente em conseguir vaga para internação, ser tratada com educação e ter cuidados técnicos com ela e o recém-nascido. $E$ que não haja diferença no atendimento desde aquelas que podem pagar por um plano de saúde para aquela com baixa renda e escolaridade.

Quanto ao acompanhamento médico durante o pré-natal, 11 entrevistadas utilizaram somente o serviço de atenção básica e duas mencionaram ter realizado o acompanhamento também em instituição privada. Essas últimas relataram ter sido influenciadas a fazer o plano de parto pela médica particular, contrapondo as demais que realizaram na unidade de saúde e, portanto, não foram influenciadas.

Mesmo a elaboração do plano de parto ser algo que já é recomendado desde 1986 não há muitos outros estudos e artigos do mesmo, tampouco informações e divulgações para a sociedade geral, tendo então poucos dados para comparações. E através da pesquisa, pode-se confirmar a baixa divulgação dessa ferramenta, pois apenas duas puérperas já ouviram falar e as demais, nunca ouviram falar em tal plano de parto, nem através dos meios decomunicação no geral e nem no momento de realização do pré-natal, mesmo todas relatando serem muito bem atendidas nele.

A não utilização do plano de parto pelas gestantes está relacionada, primeiramente ao não conhecimento dessa ferramenta, do seu proposito e benefícios e pela falta de apoio de profissionais habilitados para auxilio de sua elaboração sendo a participação deles indispensável e imprescindíveis, observa-se ainda essas lacunas e resistências apesar dos inúmeros efeitos benéficos, tendo muitos desafios e mudanças a serem enfrentados, são necessários ainda muitos estudos para ocorrer a diminuição das desigualdades entre as expectativas e as experiências vividas e o cumprimento de uma boa assistência (MEDEIROS RMK, et al, 2019).

A maior divulgação do PP, além de acarretar melhorias para a mulher, o recém-nascido e os acompanhantes, traduz-se em um forte indicador de benefícios para a saúde pública. A sua apresentação pode ajudar na redução de intervenções clínicas desnecessárias, maior preparação da gestante com seu processo de parto, maior participação, que auxilia na redução da ansiedade, medo, estresse, no momento do parto (SANTANA WN, et al., 2020).

É imprescindível instruir os profissionais enfermeiros para dar suporte as gestantes para que elas, após conhecer todo seu direito e benefícios, sejam capazes de elaborar o seu próprio plano de parto, selecionando as condutas que serão assumidas no momento do seu pré-natal, parto e pós-parto. Consequentemente mostra-se a necessidade de execução de novos estudos que cativem os enfermeiros a aderirem o uso do plano de parto na sua rotina de pré-natal para que possam favorecer tanto com a qualificação do cuidado de enfermagem, quanto com a melhor experiência de parto para as mulheres (BARROS APZ, et al., 2017).

\section{CONCLUSÃO}

Nesse estudo foi constatado de acordo com a amostra entrevistada que pouca é a divulgação do plano de parto, onde a maioria das participantes não conheciam essa ferramenta. Através de relatos das puérperas, se o plano de parto for colocado em prática ajudaria tanto para elas, em suas dúvidas, medos e desejos, quanto para os profissionais se tornando algo mais organizado, tranquilo, seguro e de formação de um grande elo de confiança entre profissionais, parturientes e familiares. Esse trabalho poderá servir como uma base e incentivo para as políticas públicas, voltadas a obstetrícia, ou seja, buscar melhorias nessa área através da conscientização dos profissionais envolvidos no pré-natal, uma vez que ele pode ser usado para mapear o conhecimento e com isso oferecer mais informações, treinamentos e divulgação dentro da saúde pública. 


\section{REFERÊNCIAS}

1. BARROS APZ, et al. Conhecimento de enfermeiras sobre plano de parto. Revista de Enfermagem da UFSM, 2017; 7 (1): 69-79.

2. CHACHAM AS, PERPÉTUO IHO. Determinantes sócio-econômicos da incidência de partos cirúrgicos em Belo Horizonte. Anais do X Encontro Nacional de Estudos Populacionais, 2016;2587-2610.

3. CORTÉS MS, et al. Uso e influência dos Planos de Parto e Nascimento no processo de parto humanizado. Rev. Latino-Am. Enfermagem, 2015;23 (3): 520-526.

4. MEDEIROS RMK, et al. Repercussões da utilização do plano de parto no processo de parturição. Ver. Gaúcha Enfermagem, 2019;40:1-12.

5. MINISTÉRIO DA SAÚDE, Gravidez, parto e nascimento com saúde, qualidade de vida e bem -estar. 2013. Disponível em: http://bvsms.saude.gov.br/bvs/publicacoes/gravidez_parto_nascimento_saude_qualidade.pdf Acessado em: 2 de setembro de 2020.

6. MINISTÉRIO DA SAÚDE. Portaria n569, de $1^{\circ}$ de Junho de 2000.2000 Disponível em: http://bvsms.saude.gov.br/bvs/saudelegis/gm/2000/prt0569_01_06_2000_rep.html Acessado em:15 de setembro de 2020.

7. MOUTA RJO, et al. Plano de parto como estratégia de empoderamento feminino. Revista Baiana de Enfermagem, 2017;31 (4): 1-10.

8. RODRIGUES MS. Humanização no processo de parto e nascimento:implicações do plano de parto. Dissertação de Mestrado. Universidade Federal de Minas Gerais, 2017: 102 p.

9. ROSA ECS, et al. Avaliação do conhecimento dos profissionais da saúde com relação ao plano de parto. Revista Ensaios Pioneiros, 2020;4 (1): 25-33.

10. SANTANA WN, et al. Plano de parto como instrumento das boas práticas no parto e nascimento: revisão integrativa Revista Baiana de Enfermagem,2020;33 (1): e32894.

11. SANTOS FSR, et al. Os significados e sentidos do plano de parto para as mulheres que participaram da Exposição Sentidos do Nascer. Cadernos de Saúde Pública, 2019;35 (6): e00143718.

12. SANTOS MLD, et al. Plano de Parto: o conhecimento da gestante sobre esta ferramenta para empoderamento durante a assistência obstétrica. Brazilian Journal of Health Review, 2020;3 (4): 10143-10165.

13. SILVA ALNV, et al. Plano de parto: ferramenta para o empoderamento de mulheres durante a assistência de enfermagem. Revista de enfermagem UFSM, 2017;07 (1): 144-151.

14. SILVA SG, et al. Perfil de gestantes participantes de rodas de conversa sobre o plano de parto. Enfermagem Obstétrica, 2015;2 (1): 9-14.

15. TORRES KN, ABI RACHED CD. A importância da elaboração do plano de parto e seus benefícios. International Journal of Health Management Review, 2017;3 (2): 1-30. 